\title{
Comparison of methods for the detection of in vitro synergy in multidrug-resistant gram-negative bacteria
}

\author{
Juliana Januario Gaudereto1, Lauro Vieira Perdigão Neto', Gleice Cristina Leite', Evelyn Patricia Sanchez Espinoza', \\ Roberta Cristina Ruedas Martins', Gladys Villas Boa Prado ${ }^{1}$, Flavia Rossi², Thais Guimarães ${ }^{1}$, Anna Sara Levin ${ }^{1}$ and \\ Silvia Figueiredo Costa ${ }^{1 *}$
}

\begin{abstract}
Background: The use of combined antibiotic therapy has become an option for infections caused by multidrugresistant (MDR) bacteria. The time-kill (TK) assay is considered the gold standard method for the evaluation of in vitro synergy, but it is a time-consuming and expensive method.

The purpose of this study was to evaluate two methods for testing in vitro antimicrobial combinations: the disk diffusion method through disk approximation (DA) and the agar gradient diffusion method via the MIC:MIC ratio. The TK assay was included as the gold standard. MDR Gram-negative clinical isolates ( $n=62 ; 28$ Pseudomonas aeruginosa, 20 Acinetobacter baumannii, and 14 Serratia marcescens) were submitted to TK, DA, and MIC:MIC ratio synergy methods.

Results: Overall, the agreement between the DA and TK assays ranged from 20 to 93\%. The isolates of $A$. baumannii showed variable results of synergism according to TK, and the calculated agreement was statistically significant in this species against fosfomycin with meropenem including colistin-resistant isolates. The MIC:MIC ratiometric agreed from 35 to $71 \%$ with TK assays. The kappa test showed good agreement for the combination of colistin with amikacin $(K=0.58 ; P=0.04)$ among the colistin-resistant $A$. baumannii isolates.
\end{abstract}

Conclusions: The DA and MIC:MIC ratiometric methods are easier to perform and might be a more viable tool for clinical microbiology laboratories.

Keywords: Synergy, Time-kill, Disk approximation, MIC:MIC ratio, Gram-negative, Multidrug-resistant

\section{Background}

Infections with multidrug-resistant (MDR) bacteria have increased dramatically over the last decade and are a major global challenge [1]. The development of new antimicrobial agents has not kept up with the emergence of new mechanisms of antibiotic resistance [2]. Moreover, inappropriate

\footnotetext{
*Correspondence: silvia.costa@pq.cnpq.br; silviacosta@usp.br

'Laboratório de Investigação Médica 49 - LIM-49, Instituto de Medicina Tropical, Hospital das Clínicas, Faculdade de Medicina, Universidade de São Paulo, Avenida Doutor Enéas de Carvalho Aguiar, 470, São Paulo, SP 05403-000, Brazil

Full list of author information is available at the end of the article
}

initial antimicrobial therapy against MDR pathogens is associated with adverse outcomes. Therefore, reducing the turnaround time while testing antimicrobial efficacy including combinations of antibiotics can lead to significant reductions in patient morbidity, mortality, and cost.

Combined antimicrobial therapy is a promising strategy for treating infections caused by MDR pathogens and can further extend antimicrobial lifespan and minimize the evolution of resistance [3, 4]. However, despite the importance of in vitro testing, methods that are accessible to

(c) The Author(s). 2020 Open Access This article is licensed under a Creative Commons Attribution 4.0 International License, which permits use, sharing, adaptation, distribution and reproduction in any medium or format, as long as you give appropriate credit to the original author(s) and the source, provide a link to the Creative Commons licence, and indicate if changes were made. The images or other third party material in this article are included in the article's Creative Commons licence, unless indicated otherwise in a credit line to the material. If material is not included in the article's Creative Commons licence and your intended use is not permitted by statutory regulation or exceeds the permitted use, you will need to obtain permission directly from the copyright holder. To view a copy of this licence, visit http://creativecommons.org/licenses/by/4.0/. The Creative Commons Public Domain Dedication waiver (http://creativecommons.org/publicdomain/zero/1.0/) applies to the data made available in this article, unless otherwise stated in a credit line to the data. 
clinical microbiology laboratories for testing synergism in a clinically actionable period are not available.

Existing methods have several disadvantages including the time-kill assay (TK), which-although considered the gold standard for synergism evaluation-is very timeconsuming and requires high technical skills $[5,6]$. The disk and epsilometer tape diffusion methods using commercially available materials are less technically demanding than the TK assay but require further validation. In this study, we evaluated two in vitro antimicrobial combination methods as alternatives to the TK method in clinical microbiology laboratories.

\section{Results}

All $A$. baumannii isolates were resistant to meropenem (MIC ranging from 16 to $128 \mu \mathrm{g} / \mathrm{mL}$ ) and fosfomycin (MIC ranged from 64 to $128 \mu \mathrm{g} / \mathrm{mL}$ ); 19/20 (95\%) were resistant to amikacin. Pseudomonas aeruginosa isolates were susceptible to colistin and resistant to meropenem (MIC ranged from 16 to $512 \mu \mathrm{g} / \mathrm{mL}$ ). Resistance to amikacin was observed in 64\% (18/28) of the Pseudomonas aeruginosa isolates. Resistance to carbapenems was found in $86 \%$ (12/ 14) of $S$. marcescens isolates, and 64\% (9/14) were resistant to amikacin. The results of sequence type (ST) and antimicrobial resistance genes from each isolate are summarized in Table 1.

The TK assay showed that $A$. baumannii isolates had synergistic effects with colistin combinations. Agreement with the DA method was $86 \%$ among the seven colistinresistant isolates except for the combination of colistin with amikacin, which showed an agreement of $14 \%$. The combination of fosfomycin with meropenem showed 50\% synergistic effects via time-kill for $A$. baumannii. The agreement between DA and TK was considered good $(\mathrm{k}=0.60$; $P=0.003)$, and the correlation was good among the seven colistin-resistant isolates $(\mathrm{k}=0.72 ; P=0.02)$ (Table 2).

The TK assay for $P$. aeruginosa isolates showed a synergistic effect for the combination of colistin + meropenem 43\% (12/28) and meropenem + amikacin 36\% (10/ 28). No synergistic effects were seen via the DA method. Agreement with TK for colistin with meropenem, colistin with amikacin, and meropenem with amikacin was 57, 93, and $64 \%$ respectively. The kappa test showed poor agreement for all of these combinations (Table 2).

The combination of colistin with meropenem had synergy against only one isolate of $S$. marcescens using the TK assay. Moreover, this isolate did not have a synergistic effect by DA. The DA and TK methods were concordant in 64, 93, and 86\% with TK for colistin with meropenem, colistin with amikacin, and ertapenem with meropenem, respectively. The DA method showed poor agreement with the TK assay via the kappa test (Table 2). Overall, the agreement between the MIC:MIC ratio method and TK assays ranged from 35 to $71 \%$ (Table 2).
The kappa test agreed well with colistin/amikacin $(\mathrm{K}=$ $0.58 ; P=0.04)$ among the seven colistin-resistant $A$. baumannii isolates.

The MIC:MIC ratio method did not identify synergistic effects for $P$. aeruginosa isolates, and the Kappa results were not statistically significant. Synergistic effects were observed for one S. marcescens isolate in both DA and MIC:MIC methods; this isolate showed no synergistic effect in $0.5 \times$ MIC (sub-inhibitory concentration) by TK assay. The agreement between the TK and MIC:MIC ratiometric methods was poor for the combination of ertapenem with meropenem. No antagonistic effects were noted.

\section{Discussion}

Multidrug-resistant Gram-negative strains such as $A$. baumannii, $P$. aeruginosa, and $S$. marcescens are commonly studied due to their major role as nosocomial pathogens with frequent development of multidrug resistance [7-13]. The treatment of infections caused by these microorganisms based on identification of resistance mechanisms and drug combinations is usually more effective than empirical treatment [1,4]. Synergy testing can also correlate to a particular resistance mechanism $[8,14]$. Such correlations might help predict the synergism for a particular antimicrobial combination for treatment. Thus, determining the molecular mechanisms can improve therapeutic outcomes.

While the rapid detection of resistance mechanisms is performed in several healthcare centers, antimicrobial interaction tests are still not available due to the challenges associated with routine combination tests. The method described here can be useful in selecting the proper combinations of antibiotics. Here, accurate and prompt treatment has an important effect on the reduction of morbidity, mortality, and costs.

Several methods have been assessed to evaluate the synergistic activity of two or more antimicrobial agents [5, 6, 15-18]. As the gold standard, the TK method yields high concordance between various studies because it produces dynamic and longitudinal information about bacterial death, which is not provided by other methods. However, the TK method is a complex technique that is difficult to perform in routine laboratories. On the other hand, antimicrobial disk methods are affordable and simple, but there are limited data evaluating the synergism of this technique in vitro with controversial results $[15,17,18]$.

In the present study, the agreement between the TK assay and the DA method was higher among colistin-resistant $A$. baumannii isolates when colistin was combined with meropenem or Fosfomycin similar to other studies [7, 9-14]. The synergistic effect of fosfomycin with carbapenem could be explained by the resistance profile of the strains evaluated-most $A$. baumannii isolates carried carbapenemase genes (bla $a_{\text {OXA-23, }}, b l a_{\text {OXA-143 }}$, and fosA), which confer 
Table 1 Sequence type according to MLST Oxford and antimicrobial resistance genes determined by PCR and WGS for 20 A. baumannii, 28 P. aeruginosa, and 14 S. marcescens

ID Sequence Antimicrobial resistance genes
Type
Acinetobacter baumannii

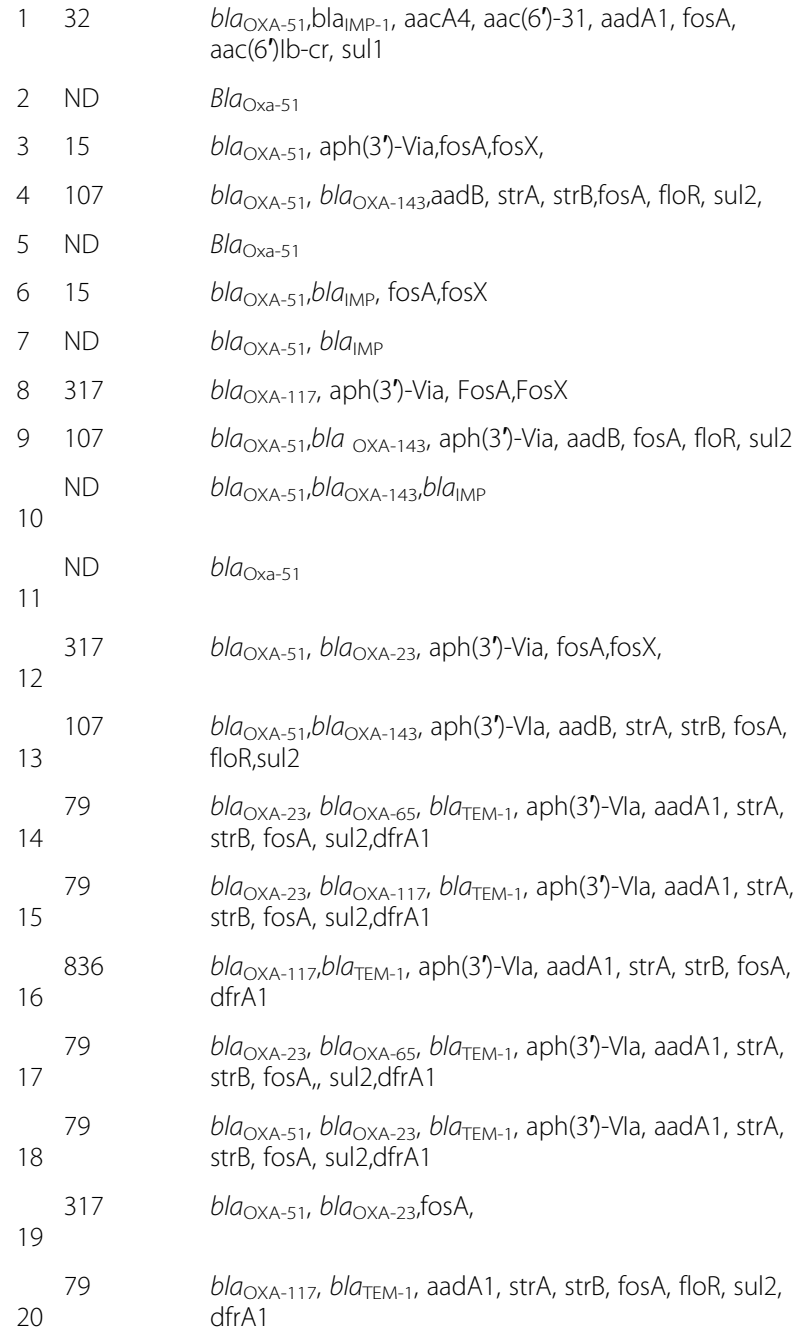

\section{Pseudomonas aeruginosa}

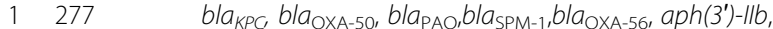

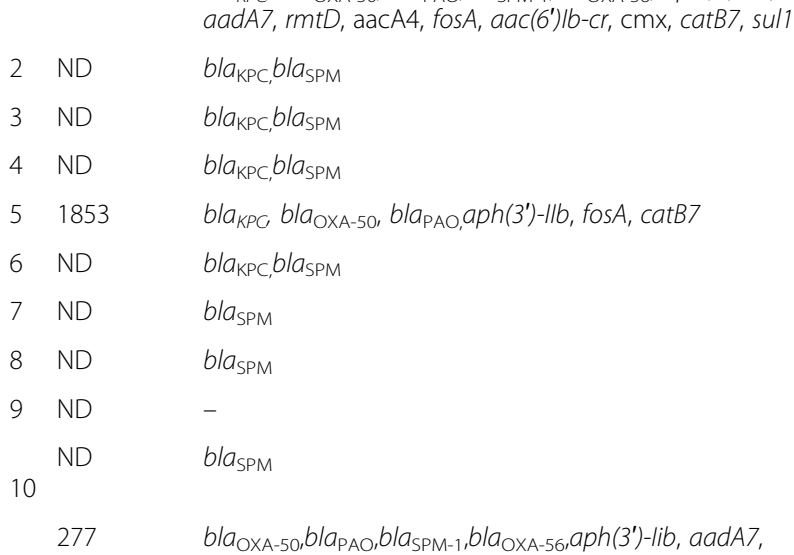

Table 1 Sequence type according to MLST Oxford and antimicrobial resistance genes determined by PCR and WGS for 20 A. baumannii, 28 P. aeruginosa, and 14 S. marcescens (Continued)

\begin{tabular}{|c|c|c|}
\hline ID & $\begin{array}{l}\text { Sequence } \\
\text { Type }\end{array}$ & Antimicrobial resistance genes \\
\hline 11 & & $a a c A 4, \operatorname{aac}\left(6^{\prime}\right) \mid b-c r, c m x, c a t B 8$ \\
\hline 12 & ND & $b l a_{\mathrm{SPM}}$ \\
\hline 13 & $\mathrm{ND}$ & $b l a_{\mathrm{SPM}}$ \\
\hline 14 & 277 & 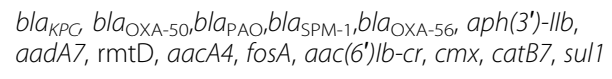 \\
\hline 15 & ND & - \\
\hline 16 & ND & - \\
\hline 17 & ND & bla $a_{\mathrm{SPM}}$ \\
\hline 18 & ND & $b l a_{\mathrm{SPM}}$ \\
\hline 19 & ND & $b l a_{\mathrm{SPM}}$ \\
\hline 20 & 277 & 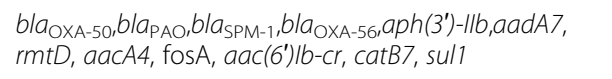 \\
\hline 21 & ND & $b l a_{S P M}$ \\
\hline 22 & ND & $b l a_{\mathrm{SPM}}$ \\
\hline 23 & ND & - \\
\hline 24 & ND & bla $a_{\mathrm{SPM}}$ \\
\hline 25 & ND & bla $a_{\mathrm{SPM}}$ \\
\hline 26 & ND & - \\
\hline 27 & ND & $b / a_{\mathrm{SPM}}$ \\
\hline 28 & ND & $b l a_{\mathrm{SPM}}$ \\
\hline
\end{tabular}

\section{Serratia marcescens}

1 NA bla $a_{\mathrm{SRT}-2,}, b a_{\mathrm{KPC}-2}, a p h\left(3^{\prime}\right)-\mathrm{Vla}, \operatorname{aacA4}, \operatorname{aac}\left(6^{\prime}\right)-1 \mathrm{c}$, ant (2")-la, aac (6')-lb-cr, cat (pC194), sul2, dfrA8

2 NA bla $a_{\mathrm{SRT}-2}, b a_{\mathrm{KPC}-2}, a p h\left(3^{\prime}\right)-\mathrm{Vla}, \operatorname{aacA4}, \operatorname{aac}\left(6^{\prime}\right)-\mathrm{lc}$, ant $\left(2^{\prime \prime}\right)-1 a, a a c\left(6^{\prime}\right)-1 b-c r$, sul2, dfrA8

3 NA bla $a_{\mathrm{SRT}-2,}, b a_{\mathrm{KPC}-2}, a p h\left(3^{\prime}\right)-\mathrm{Vla}, \operatorname{aacA4}, \operatorname{aac}\left(6^{\prime}\right)-\mathrm{lc}$, ant (2")-la, aac(6')-lb-cr, cat (pC194), sul2

4 NA bla $a_{\mathrm{SRT}-2}, b a_{\mathrm{KPC}-2,}, a p h\left(3^{\prime}\right)-\mathrm{Vla}, \operatorname{aacA4}, \operatorname{aac}\left(6^{\prime}\right)-/ c$, ant(2")-la, aac (6')-lb-cr,sul2, dfrA8

5 NA bla $a_{\mathrm{SRT}-2,}, b / a_{\mathrm{KPC}-2}, a p h\left(3^{\prime}\right)$-Vla, $\operatorname{aacA4}, \operatorname{aac}\left(6^{\prime}\right)-\mathrm{lc}$, ant (2")-la, aac (6')-lb-cr, sul2, dfrA8

6 NA bla $a_{\mathrm{SRT}-2,}, \mathrm{bla}_{\mathrm{KPC}-2}, \operatorname{aph}\left(3^{\prime}\right)$-Vla, aacA4,aac $\left(6^{\prime}\right)-\mathrm{lc}$ ant (2")-la, aac (6')-lb-cr, sul2, dfrA8 
Table 1 Sequence type according to MLST Oxford and antimicrobial resistance genes determined by PCR and WGS for 20 A. baumannii, 28 P. aeruginosa, and 14 S. marcescens (Continued)

\begin{tabular}{|c|c|c|}
\hline ID & $\begin{array}{l}\text { Sequence } \\
\text { Type }\end{array}$ & Antimicrobial resistance genes \\
\hline & & sult, dfrA22 \\
\hline 8 & NA & 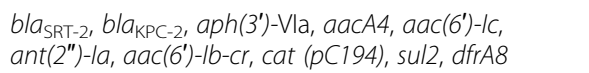 \\
\hline 9 & NA & $\begin{array}{l}\text { bla } a_{\text {SRT-2, }} \text { aacA4, aac }\left(6^{\prime}\right)-1 c, \text { ant }\left(2^{\prime \prime}\right)-1 a, \text { aac }\left(6^{\prime}\right)-1 b-c r \text {, } \\
\text { sul2, dfrA8 }\end{array}$ \\
\hline 10 & NA & $\begin{array}{l}\text { bla } a_{\mathrm{SRT}-2,}, b / a_{\mathrm{KPC}-2}, a a c A 4, a a c\left(6^{\prime}\right)-1 c \text {, ant }\left(2^{\prime \prime}\right)-1 a, \\
\text { acc }\left(6^{\prime}\right)-1 b-c r, \text { sul2, dfrA8 }\end{array}$ \\
\hline 11 & NA & $\begin{array}{l}\text { bla } a_{\mathrm{SRT}-2,}, b / a_{\mathrm{KPC}-2,}, b / a_{\mathrm{SHV}-5}, a a c A 4, \operatorname{aac}\left(6^{\prime}\right)-c_{1} \\
\text { ant }\left(2^{\prime \prime}\right)-1 a, \text { sul2, dfrA }\end{array}$ \\
\hline 12 & NA & - \\
\hline 13 & NA & 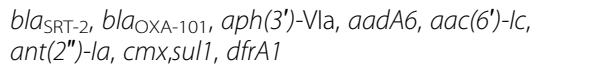 \\
\hline 14 & NA & $\begin{array}{l}\text { bla } a_{\mathrm{SRT}-2,}, b a_{\mathrm{KPC}-2,} \text {, bla } a_{\mathrm{SHV}-5}, a a c A 4, \operatorname{aac}\left(6^{\prime}\right)-\mathrm{lc} \text {, ant (2")-Ia, } \\
\operatorname{aac}\left(6^{\prime}\right)-1 b-c r, \text { sul2 }\end{array}$ \\
\hline
\end{tabular}

ID Isolates; ND not WGS; NA not applicable

resistance to fosfomycin. Both classes of antimicrobials act at different sites of the bacterial cell wall and inhibit cell wall synthesis [7, 9]. Perdigão-Neto et al. [19] also demonstrated that fosfomycin is a promising drug-particularly in combination with meropenem for the treatment of infections due to pan-resistant Gram-negative bacteria.

Nevertheless, the in vitro synergy effect of colistin against $S$. marcescens is controversial $[11,16]$. The synergistic effect appears lower for combinations with colistin among $S$. marcescens isolates and species that exhibit intrinsic resistance to polymyxins [14]. In our study, we found a lower synergistic effect against $S$. marcescens isolates in colistin combinations consistent with Nastro [11] who evaluated colistin with rifampicin against colistinresistant $A$. baumannii, K. pneumoniae, and S. marcescens isolates by E-test/agar dilution and TK assays. This work noted synergy for all isolates except two of five $S$. marcescens isolates. Thus, it seems that combinations using colistin are not useful for treating Serratia infections.

Few studies have compared the MIC:MIC ratio and the TK assay against a large collection of MDR bacteria $[6,20-22]$. One of the largest studies [6] compared three E-test methods with TK against $31 \mathrm{KPC}$-producing Klebsiella isolates. The MIC:MIC ratio showed a better correlation with the TK assay: concordance of $80.6 \%$ and a significant Kappa value of $0.59(P<0.001)$. In our study, the agreement was statistically significant only for the combination of colistin with amikacin $(\mathrm{K}=0.58 ; P=$ 0.04 ) among the colistin-resistant $A$. baumannii isolates.

The agreement between MIC:MIC ratio and TK assays ranged from 35 to $71 \%$, which is similar to those described by Chachanidze et al. [23] who compared the results of TK with MIC:MIC ratio for 31 fluoroquinoloneresistant $P$. aeruginosa isolates. These authors evaluated a combination of levofloxacin and piperacillin/tazobactam and found 77\% agreement between the methods. Some studies have shown clinical application of synergism [19, 24]. Perdigão Neto et al. showed better clinical outcomes in Gram-negative infections treated with combined therapy with known in vitro synergism [19]. In addition, some studies have shown the benefit of combined therapy despite the resistance of the microorganism to some antimicrobials in the scheme [24].

Table 2 Concordance analysis data among disk approximation and MIC:MIC ratio methods with time-kill assay according to antimicrobial combinations tested for A. baumannii, P. aeruginosa, and S. marcescens

\begin{tabular}{|c|c|c|c|c|}
\hline \multirow{2}{*}{$\begin{array}{l}\text { Drugs combination/Species } \\
\text { Acinetobacter baumannii }(\boldsymbol{n}=\mathbf{2 0})\end{array}$} & \multicolumn{2}{|c|}{ Disk approximation and TK } & \multicolumn{2}{|c|}{ MIC:MIC ratio and TK } \\
\hline & Concordance (\%) & Kappa/p value & Concordance (\%) & Kappa/p value \\
\hline Colistin with meropenem & 30 & $0.0 / 0.500$ & 40 & 0.0 \\
\hline Colistin with amikacin & 20 & $0.0 / 0.333$ & 35 & $0.04 / 0.25$ \\
\hline Colistin with fosfomycin & 30 & $0.0 / 0.500$ & NA & NA \\
\hline Fosfomycin with meropenem & 80 & $0.60 / 0.003$ & NA & NA \\
\hline Fosfomycin with gentamycin & 75 & $-0.0 / 0.696$ & NA & NA \\
\hline \multicolumn{5}{|l|}{ Pseudomonas aeruginosa $(n=28)$} \\
\hline Colistin with meropenem & 57 & 0.0 & 57 & 0.0 \\
\hline Colistin with amikacin & 93 & 0.0 & 68 & 0.0 \\
\hline Meropenem with amikacin & 64 & 0.0 & NA & NA \\
\hline \multicolumn{5}{|l|}{ Serratia marcescens $(n=14)$} \\
\hline Colistin with meropenem & 64 & $-0.12 / 0.744$ & NA & NA \\
\hline Colistin with amikacin & 93 & 0.0 & NA & NA \\
\hline Ertapenem with meropenem & 86 & $0.0 / 0.500$ & 71 & $0.25 / 0.081$ \\
\hline
\end{tabular}


Synergism has also been described using DA and MIC: MIC methods against Gram-positive organisms-especially S. aureus [25-27]; these are likely useful methods against Gram-positive organisms. Unfortunately, for logistical reasons, we could not evaluate in vitro synergy against Gram-positive standard organisms. This is an important limitation of our study. We evaluated A. baumannii belonging to international STs such as ST15 and ST297 [28, 29] as well as $S$. marcescens carrying KPC-the most frequent carbapenemase described for this organism $[1,2]$. Thus, our findings can be useful internationally.

There are some important limitations to this study. First, we tested a relatively small number of isolates from only one hospital and the $P$. aeruginosa isolates belong to the endemic clone ST277 isolated mainly in Brazil [30]. However, the isolates evaluated were identified during an 11year period and have been well-characterized (phenotypically and genotypically). Therefore, our findings indicate that DA and MIC:MIC ratio methods can be useful to help infectious disease clinicians handle infections caused by carbapenem-resistant organisms. DA and MIC:MIC methods can highlight the in vitro synergy and avoid combination therapies that will increase cost and side effects.

\section{Conclusions}

We found that the DA method has good agreement with the TK assay for Fosfomycin/meropenem combinations against colistin-resistant $A$. baumannii isolates carrying carbapenemases and fosA genes. The feasibility of the DA method depends on the bacterial resistance mechanism. The DA and MIC:MIC ratio methods are easy to perform and are suitable for the screening of synergy in clinical microbiology laboratories. Further studies are needed to evaluate these methods against a large collection of organisms including Gram-positive bacteria.

\section{Methods}

\section{Bacterial isolates}

We selected 62 clinical isolates of MDR Gram-negative bacteria studied previously [3]. This cohort included 20 A. baumannii isolates identified from 2002 to 2012; 28 P. aeruginosa isolates from 2011 to 2013; and 14 S. marcescens isolates from 2010 to 2013. All samples were collected from patients at the Hospital das Clínicas da Universidade de São Paulo (HC-FMUSP). Identification was performed using an automated Vitek 2 system (BioMérieux, Hazelwood, MO). All non-fermenting isolates and $86 \%$ of Enterobacteria were carbapenem-resistant; seven isolates of $A$. baumannii were colistin-resistant.

\section{Genotypic characterization}

Carbapenemases genes (bla $a_{\text {OXA-23-like, }} b l a_{\text {OXA-51-like, }}$ bla $a_{\mathrm{OXA}-58-\text {-like, }}, b l a_{\mathrm{OXA}-24-\mathrm{like}}, b l a_{\mathrm{IMP}}, b_{\mathrm{SPM}}, b l a_{\mathrm{VIM}}, b l a_{\mathrm{SIM}}$, $b l a_{\mathrm{NDM}}, b l a_{\mathrm{OXA}-143-\text { like, }}$ and $\left.b l a_{\mathrm{KPC}}\right)$ were investigated by
PCR [31-33], and the other genes were studied by wholegenome sequence (WGS). Thirty-four isolates (16 A. baumannii, $13 S$. marcescens, and 5 P. aeruginosa) were characterized by WGS using MiSeqIllumina ${ }^{\text {Tw }}$ technology. The files were analyzed by VelvetOptimizer v.2.2.5 software (Victorian Bioinformatics Consortium, Australia). Genome annotation was performed using Prokka [34]. The resistance genes were investigated using Resfinder (https:// cge.cbs.dtu.dk). The sequence type (ST) was determined by MLSTfinder (Multilocus Sequence Typing) [35].

\section{Antimicrobial sensitivity test}

The minimal inhibitory concentrations (MIC) of colistin, meropenem (USP Reference Standard, Rockville, MD, USA), gentamicin, amikacin, and ertapenem (Sigma - Aldrich, St Louis, MO, USA) were determined via the broth microdilution method, and fosfomycin was determined (Sigma - Aldrich, St Louis, MO, USA) via the goldstandard agar dilution method. The assays were performed in duplicate on alternate days as recommended by the Clinical and Laboratory Standards Institute (CLSI) [36]; samples were quality control tested with $P$. aeruginosa ATCC 27853 and E. coli ATCC 25922 strains. CLSIrecommended breakpoints were used for all antimicrobials except for colistin and fosfomycin for which European Committee on Antimicrobial Susceptibility Testing (EUCAST) breakpoints were used including the breakpoints for fosfomycin in Enterobacteriaceae for the categorization of the A. baumannii isolates [37].

\section{Synergy tests}

To identify synergistic effects, the TK, disk approximation, and MIC:MIC ratio methods were performed in duplicate. Each antimicrobial combination was chosen as previously described in the literature and the treatment options available in our hospital. For the MIC:MIC ratio, we tested the susceptibility of non-fermenting Gramnegative isolates against colistin with meropenem and colistin with amikacin combination as well as the susceptibility of Enterobacteria isolates against ertapenem with meropenem.

\section{Time-kill method}

The TK method was performed as previously described [38]. Antimicrobials were tested alone and in combination with concentrations ranging from $1 \times$ to $0.5 \times$ MIC. Control experiments without antimicrobial agents were conducted simultaneously with the TK assay. The vials containing cation-adjusted Mueller-Hinton broth, antimicrobials, and the tested organisms at an initial density of $10^{6} \mathrm{CFU} / \mathrm{ml}$ (10 $\mathrm{ml}$ volume) were incubated at $35 \pm 2{ }^{\circ} \mathrm{C}$ in ambient air. Aliquots were removed at 0, 2, 4, 6, and $24 \mathrm{~h}$ and serially diluted in $0.9 \%$ sodium chloride solution and plated on Mueller-Hinton agar plates for viable-colony 
counting. The synergy effect was defined as $\mathrm{a} \geq 2 \log 10$ $\mathrm{CFU} / \mathrm{ml}$ reduction in colony counting when compared to the most active single agent after incubation for $24 \mathrm{~h}$. The antagonism was defined by an increase of $\geq 2 \log 10$ CFU/ml in the combination versus the most active single agent. The no difference (ND) effect was established as an increase or decrease of $<2 \log 10$ in colony counting with an antibiotic combination versus individual antimicrobials [38].

\section{Disk approximation (DA)}

Commercial disks were purchased from Oxoid ${ }^{\bullet}$ (Basingstoke, UK) including colistin $(10 \mu \mathrm{g})$, amikacin $(30 \mu \mathrm{g})$, gentamicin $(10 \mu \mathrm{g})$, meropenem $(10 \mu \mathrm{g})$, ertapenem $(10 \mu \mathrm{g})$, fosfomycin $(200 \mu \mathrm{g})$, and gentamicin $(10 \mu \mathrm{g})$. These were placed $5 \mathrm{~mm}$ apart on $150-\mathrm{mm}$ diameter Mueller-Hinton agar plates cultured with organisms adjusted to the 0.5 McFarland standard and incubated at $35 \pm 2{ }^{\circ} \mathrm{C}$ for 16 to $18 \mathrm{~h}$ [39]. Synergism was defined by inhibition zone bridging. Antagonism was indicated by truncation of the inhibition zone at the junction of the antimicrobials; ND was defined as the formation of two independent circles around the antibiotic disks [15].

\section{MIC:MIC ratio}

The MIC was initially determined using strips impregnated with colistin (bioMérieux, France), amikacin, meropenem (Thermo Fisher Scientific, Basingstoke, UK), and ertapenem (Liofilchem, Italy) at increasing concentrations. For the synergism testing, one test strip was placed on the inoculated MHA plate. After $1 \mathrm{~h}$ at room temperature, the agar was marked adjacent to the previously determined MIC of the agent, and the tape was replaced. The second strip was then placed over the imprint of the previous strip such that the mark on the agar corresponds to the MIC of the second agent [20]. The highest value was considered for isolates in which the MIC exceeded the value of the strip concentration [6]. The resulting ellipse of inhibition was checked after $18-20 \mathrm{~h}$ at $35 \pm 2{ }^{\circ} \mathrm{C}$ and the Fractional Inhibitory Concentration Index ( $\mathrm{FFIC}$ ) was calculated and interpreted using the following criteria: synergism, $\Sigma F I C \leq 0.5$; antagonism, $\Sigma F I C>4$; and indifferent, $\Sigma F I C>0.5-4[6,15,20,31-40]$.

\section{Analysis of the results of synergy tests}

The Kappa statistical test was performed using STATA software (College Station, TX, USA) version 13. The results of the DA and MIC:MIC ratio were compared to the TK results to establish the gold standard. The concentration of $1 \times$ MIC was chosen for comparison of the TK with the MIC:MIC ratio because this concentration used an epsilometric test [6]. The results were interpreted as poor agreement when $\mathrm{k}<0.40$; good agreement when $\mathrm{k}=0.40-0.75$; and very good agreement when $\mathrm{k}>0.75$ [40]. $P$-values < 0.05 was considered statistically significant. The correlation was calculated by a ratio of concordant responses among the evaluated methods.

\section{Abbreviations}

CLSI: Clinical and Laboratory Standards Institute; DA: Disk approximation; EUCAST: European Committee on Antimicrobial Susceptibility Testing; FIC: Fractional inhibitory concentration; MDR: Multidrug-resistant; MIC: Minimal inhibitory concentration; TK: Time-kill; WGS: whole-genome sequence

\section{Authors' contributions}

JJG assembled the data and drafted the manuscript. GCL, GVLP, and ES helped in MIC determination and synergy; RCR was responsible for wholegenome sequencing and bioinformatics analysis; LVPN, FR, TG, and ASL helped with the draft of manuscript; and SFC designed, supervised, and assessed the study and drafted the manuscript. All authors have read, contributed, and approved the final manuscript.

\section{Funding}

Internal funding from the University of São Paulo, Brazil, and the National Council of Technological and Scientific Development (CNPQ), Brazil supported this study. The author Juliana Januario Gaudereto received a scholarship from CNPQ.

\section{Availability of data and materials}

The datasets used and/or analysed during the current study available from the corresponding author on reasonable request.

\section{Ethics approval and consent to participate}

All data were analysed anonymously and confidentially, with approval by the Research Ethics Committee of Hospital das Clinicas of the University of Sao Paulo and received approval by CONEP (National Ethics Commission), Brazil. It was a retrospective in vitro study, thus, it was not possible to apply the consent to participate.

\section{Consent for publication}

Not applicable.

\section{Competing interests}

The authors declare that they have no competing interests.

\section{Author details}

'Laboratório de Investigação Médica 49 - LIM-49, Instituto de Medicina Tropical, Hospital das Clínicas, Faculdade de Medicina, Universidade de São Paulo, Avenida Doutor Enéas de Carvalho Aguiar, 470, São Paulo, SP 05403-000, Brazil. ²Divisão de Laboratório Central - Serviço de Microbiologia Clínica, Hospital das Clínicas, Faculdade de Medicina, Universidade de São Paulo, São Paulo, SP, Brazil.

Received: 23 July 2019 Accepted: 20 March 2020

Published online: 16 April 2020

\section{References}

1. Giamarellou H. Multidrug-resistant gram-negative bacteria: how to treat and for how long. Int J Antimicrob Agents. 2010;36S:S50-4.

2. Logan LK, Weinstein RA. The epidemiology of carbapenem-resistant Enterobacteriaceae: the impact and evolution of a global menace. J Infect Dis. 2017;215(Suppl1):S28-36

3. Leite GC, Oliveira MS, Perdigão-Neto LV, Rocha CKD, Guimarães T, Rizek C, et al. Antimicrobial combinations against pan-resistant Acinetobacter baumannii isolates with different resistance mechanisms. PLoS ONE. 2016; 11(3):e0151270.

4. Sharma R, Patel S, Abboud C, Diep J, Ly NS, Pogue JM, Kaye KS, Li J, Rao GG. Polymyxin $B$ in combination with meropenem against carbapenemaseproducing Klebsiella pneumoniae: pharmacodynamics and morphological changes. Int J Antimicrob Agents. 2017;49:224-32.

5. Sopirala MM, Mangino JE, Gebreyes WA, Biller B, Bannerman T, Balada-Llasat JM, Pancholi P. Synergy testing by Etest, microdilution checkerboard, and time-kill methods for pan-drug-resistant a Acinetobacter baumannii. Antimicrob Agents Chemother. 2010;54:4678-83. 
6. Pankey GA, Ashcraft DS, Dornelles A. Comparison of 3 Etest ${ }^{\oplus}$ methods and time-kill assay for determination of antimicrobial synergy against carbapenemase-producing Klebsiella species. Diagn Microbiol Infect Dis. 2013;77:220-6.

7. Samonis G, Maraki S, Karageorgopoulos DE, Vouloumanou EK, Falagas ME. Synergy of fosfomycin with carbapenems, colistin, netilmicin, and tigecycline against multidrug-resistant Klebsiella pneumoniae, Escherichia coli, and Pseudomonas aeruginosa clinical isolates. Eur J Clin Microbiol Infect Dis. 2012;31:695-701.

8. Evren E, Azap OK, Çolakoğlu S, Arslan H. In vitro activity of fosfomycin in combination with imipenem, meropenem, colistin and tigecycline against OXA 48-positive Klebsiella pneumoniae strains. Diagn Microbiol Infect Dis. 2013;76:335-8.

9. Singkham-in U, Chatsuwan T. In vitro activities of carbapenems in combination with amikacin, colistin, or fosfomycin against carbapenemresistant Acinetobacter baumannii clinical isolates. Diagn Microbiol Infect Dis. 2018:91:169-17.

10. Gaibani P, Lombardo D, Lewis RE, Mercuri M, Bonora S, Landini MP, Ambretti S. In vitro activity and post-antibiotic effects of colistin in combination with other antimicrobials against colistin-resistant KPCproducing Klebsiella pneumoniae bloodstream isolates. J Antimicrob Chemother. 2014;69:1856-65.

11. Nastro M, Rodriguez $\mathrm{CH}$, Monge R, Zintgraff J, Neira L, Rebollo M, Vay C, Famiglietti A. Activity of the colistin-rifampicin combination against colistinresistant, carbapenemase producing gram-negative bacteria. J Chemother. 2014;26:211-6

12. Ni W, Shao X, Di X, Cui J, Wang R, Liu Y. In vitro synergy of polymyxins with other antibiotics for Acinetobacter baumannii: a systematic review and metaanalysis. Int J Antimicrob Agents. 2015;45:8-18.

13. Bae S, Kim MC, Park SJ, Kim HS, Sung H, Kim MN, Kim SH, Lee SO, Choi SH, Woo JH, Kim YS, Chong YP. In vitro synergistic activity of antimicrobial agents in combination against clinical isolates of colistin-resistant Acinetobacter baumannii. Antimicrob Agents Chemother. 2016;60:6774-9.

14. Brennan-Krohn T, Pironti A, Kirby JE. Synergistic activity of colistincontaining combinations against colistin-resistant Enterobacteriaceae. Antimicrob Agents Chemother. 2018;62:e00873-18.

15. García-Salguero C, Rodríguez-Avial I, Picazo JJ, Culebras E. Can plazomicin alone or in combination be a therapeutic option against carbapenem resistant Acinetobacter baumanni? Antimicrob.AgentsChemother. 2015;59:5959-66.

16. Ostenson RC, Fields BT, Nolan CM. Polymyxin B and rifampin: new regimen for multiresistant Serratia marcescens infections. Antimicrob Agents Chemother. 1977;12:655-9.

17. Montanari MP, Piccolib I, Mingoiaa M, Marchettib F, Varaldoa PE. Synergistic potential of ceftazidime plus amikacin or levofloxacin against Pseudomonas aeruginosa as determined using a checkerboard and a disk diffusion technique. Diagn Microbiol Infect Dis. 2005;53:157-60.

18. Stein C, Makarewicz O, Bohnert JA, Pfeifer Y, Kesselmeier M, Hagel S, Pletz MW. Three dimensional checkerboard synergy analysis of Colistin, Meropenem, Tigecycline against multidrug-resistant clinical Klebsiella pneumonia isolates. PLoSONE. 2015;10(6):e0126479.

19. Perdigão Neto LV, Oliveira MS, Martins RCR, Marchi AP, Gaudereto JJ, da Costa LATJ, de Lima LFA, Takeda CFV, Costa SF, Levin AS. Fosfomycin in severe infections due to genetically distinct pan-drug-resistant gramnegative microorganisms: synergy with meropenem. J Antimicrob Chemother. 2019;74(1):177-81.

20. Pankey GA, Ashcraft DS. In vitro synergy of ciprofloxacin and gatifloxacin against ciprofloxacin-resistant Pseudomonas aeruginosa. Antimicrob Agents Chemother. 2005:49:2959-64.

21. Pankey GA, Ashcraft DS. The detection of synergy between meropenem and polymyxin B against meropenem-resistant Acinetobacter baumannii using Etest ${ }^{\oplus}$ and time-kill assay. Diagn Microbiol Infect Dis. 2009;63:228-32.

22. Pankey GA, Ashcraft DS. Detection of synergy using the combination of polymyxin B with either meropenem or rifampin against carbapenemaseproducing Klebsiella pneumoniae. Diagn Microbiol Infect Dis. 2011;70:561-4.

23. Chachanidze V, Curbelo-lrizarry A, Ashcraft D, Pankey G. In vitro synergy of levofloxacin plus piperacillin-tazobactam against Pseudomonas aeruginosa. Interdiscip Perspect Infect Dis. 2009;984934.

24. Tumbarello M, Trecarichi EM, De Rosa FG, Giannella M, Giacobbe DR, Bassetti $\mathrm{M}$, et al. Infections caused by KPC-producing Klebsiella pneumoniae: differences in therapy and mortality in a multicentre study. J Antimicrob Chemother. 2015;70:2133-43.
25. Sakoulas G, Olson J, Yim J, Singh NB, Kumaraswamy M, Quach DT, Rybak MJ Pogliano J, Nizet V. Cefazolin and Ertapenem, a synergistic combination used to clear persistent Staphylococcus aureus bacteremia. Antimicrob Agents Chemother. 2016;60(11):6609-18.

26. Brochmann RP, Helmfrid A, Jana B, Magnowska Z, Guardabassi L. Antimicrobial synergy between carprofen and doxycycline against methicillin-resistant Staphylococcus pseudintermedius ST71. BMC Vet Res. 2016;12(1):126.

27. Sy CL, Huang TS, Chen CS, Chen YS, Tsai HC, Wann SR, Wu KS, Chen JK, Lee SS, Liu YC. Synergy of $\beta$-lactams with Vancomycin against methicillinresistant Staphylococcus aureus: correlation of disk diffusion and checkerboard methods. J Clin Microbiol. 2016;54(3):565-8.

28. Hamidian M, Nigro SJ. Emergence, molecular mechanisms and global spread of carbapenem-resistant Acinetobacter baumannii. Microbial Genomics. 2019. https://doi.org/10.1099/mgen.0.000306.

29. Chagas TP, Carvalho KR, de Oliveira Santos IC, Carvalho-Assef AP, Asensi MD. Characterization of carbapenem-resistant Acinetobacter baumannii in Brazil (2008-2011): countrywide spread of OXA-23-producing clones (CC15 and CC79). Diagn Microbiol Infect Dis. 2014;79:468-72.

30. Silva FM, Carmo MS, Silbert S, Gales AC. SPM-1-producing Pseudomonas aeruginosa: analysis of the ancestor relationship using multilocus sequence typing, pulsed-field gel electrophoresis, and automated ribotyping. Microb Drug Resist. 2011;17:215-20.

31. Bradford PA, Bratu S, Urban C, Visalli M, Mariano N, Landman D, Rahal JJ, Brooks S, Cebular S, Quale J. Emergence of carbapenem-resistant Klebsiella species possessing the class a carbapenem-hydrolyzing KPC-2 and inhibitorresistant TEM-30 beta-lactamases in New York City. Clin Infect Dis. 2004;39: 55-60.

32. Mendes RE, Kiyota KA, Monteiro J, Castanheira M, Andrade SS, Gales AC, Pignatari AC, Tufik S. Rapid detection and identification of Metallo-betalactamase encoding genes by multiplex real time PCR assay and melt curve analysis. J Clin Microbiol. 2007;2:544-7.

33. Mostachio AK, Van der Heidjen I, Rossi F, Levin AS, Costa SF. Multiplex PCR for rapid detection of genes encoding Oxacillinases and metallo-betalactamases in colistin-susceptible Acinetobacter spp. J Med Microbio. 2009; 58:1522-4.

34. Seemann T. Prokka: rapid prokaryotic genome annotation. Bioinformatics. 2014;30:2068-9.

35. Larsen MV, Cosentino S, Rasmussen S, Friis C, Hasman H, Marvig RL, Jelsbak L, Sicheritz-Pontén T, Ussery DW, Aarestrup FM, Lund O. Multilocus sequence typing of Total genome sequenced Bacteria. J Clin Microbiol. 2012;50:1355-61.

36. Clinical and Laboratory Standards Institute. Performance standards for antimicrobial susceptibility testing (M100-S28). Wayne: CLSI; 2018.

37. European Committee on Antimicrobial Susceptibility Testing (EUCAST). Breakpoint tables for interpretation of MICs and zone diameters. Version 8.1; 2018. Available from: http://www.eucast.org/. [Accessed 10 October 2018].

38. Petersen PJ, Labthavikul P, Jones $\mathrm{CH}_{\text {, Bradford PA. In vitro antibacterial }}$ activities of tigecycline in combination with other antimicrobial agents determined by chequerboard and time-kill kinetic analysis. J Antimicrob Chemother. 2006;57:573-6.

39. Pillai SK, Moellering RC, Eliopoulos GM. Antimicrobial combinations. In: Lorian V, editor. Antibiotics in laboratory medicine. 5th ed. Philadelphia: Lippincott Williams and Wilkins; 2005. p. 365-440.

40. Fleiss J. Statistical methods for rates and proportions. 2nd ed. New York: Wiley; 1981

\section{Publisher's Note}

Springer Nature remains neutral with regard to jurisdictional claims in published maps and institutional affiliations. 